\title{
Gewinner und Verlierer von Stadtentwicklung: Ein Plädoyer für mehr Wohneigentum
}

\author{
Gabriel M. Ahlfeldt • Wolfgang Maennig
}

Online publiziert: 25. Oktober 2019

(C) Der/die Autor(en) 2019

Zusammenfassung Der Wunsch nach mehr Wohneigentum ist in Deutschland ungebrochen. Allerdings gelingt es nicht allen Haushalten, diesen Wunsch in die Tat umzusetzen.

Die theoretische und empirische Literatur zu den externen Nutzen und Kosten der Eigennutzung liefert keine überzeugenden Argumente, welche ein politisches Gegensteuern gegen steigende Eigennutzerquoten rechtfertigen. Im vorliegenden Beitrag führen wir als bisher weitgehend unbeachteten externen Nutzen der Eigennutzung an, dass die regressiven Verteilungswirkungen einer auf Verbesserung von Lebensumständen bedachten Stadtentwicklungspolitik verringert werden: Während Eigentümer von Entwicklungsmaßnahmen in ihrer Umgebung meist gewinnen, verlieren Mieter in der Regel.

Die nicht-konservativen Parteien unterstützen in Deutschland jedoch kaum die Wohneigentumsbildung; dies verwundert angesichts dessen, dass die Mehrheit der nicht-konservativen Wähler in den meisten vergleichbaren Ländern Hauseigentümer sind; in Deutschland sind 49\% der SPD-Wähler Immobilieneigentümer (Kohl 2018). Die nicht-konservativen Parteien in Deutschland sollten Strategien entwickeln, mit denen sie durch aktive ,linke Politik“ den Traum vom Eigenheim realisieren und Wähler erhalten oder sogar gewinnen können.

Schlüsselwörter Mieten · Verdrängung · Crux der Demokratie ·

Volksabstimmungen · Umverteilung · Eigenheimpolitik

\footnotetext{
G. M. Ahlfeldt ( $\triangle)$

Department of Geography and Environment, London School of Economics (LSE), Houghton Street, London, WC2A 2 AE, Großbritannien

E-Mail: g.ahlfeldt@1se.ac.uk

W. Maennig

Department of Economics, Universität Hamburg, Von-Melle-Park 5, 20146 Hamburg, Deutschland

E-Mail: wolfgang.maennig@uni-hamburg.de
} 


\section{Winners and losers of urban development: a plea for more home ownership}

Abstract The desire for more home ownership in Germany remains unbroken. However, not all households succeed in putting this desire into practice. The theoretical and empirical literature on the external benefits and costs of owner-occupancy does not provide convincing arguments to justify political countermeasures against rising owner-occupier rates. In this paper, we argue that the regressive distributional effects of an urban development policy that aims to improve living conditions may be reduced with increasing owner-occupancy: While owners of development measures usually gain from their surroundings, tenants usually lose.

However, the non-conservative parties in Germany hardly support home ownership. This is surprising given that the majority of non-conservative voters in most comparable countries are homeowners; in Germany, 49\% of SPD voters are property owners. Non-conservative parties in Germany should develop strategies to realize the dream of home ownership through active "left-wing politics" and to maintain or even win voters.

Keywords Rents · Displacement - Crux of democracy · Referendums · Redistribution · Home-ownership policy

\section{Einführung}

Die jüngst steigenden Mieten in deutschen Groß- und Universitätsstädten schüren Ängste vor Verdrängung finanziell schwächerer Milieus, in Städten wie München gar von Teilen der Mittelschicht. Diese Sorgen führen zu einer zögerlichen, wenn nicht blockierenden Haltung gegenüber potentiell aufwertenden Änderungen. Sogar Stadtentwicklungspolitiken mit offensichtlichen Verbesserungen der Lebensqualität, wie beispielsweise Verringerungen von Fluglärm werden von Anwohnern abgelehnt, sei es in niedrigschwelligen Bürgerbeteiligungsverfahren oder bei Volksabstimmungen (Ahlfeldt und Maennig 2015). Veränderungen in der Nachbarschaft, welche die Beschäftigung und Kaufkraft positiv beeinflussen können, stoßen ebenfalls auf Ablehnung. Als prominentes Beispiel scheiterte kürzlich der in Berlin-Kreuzberg geplante Google-Campus am Widerstand der Anwohner, die Sorge vor zuziehenden Google-Mitarbeitern und steigendenden Mieten hatte (Hackenbruch und Simon 2018).

Aufwertungen von Wohnlagen haben offensichtlich Umverteilungswirkung - zu Lasten von Mietern, und zu Gunsten von Vermietern. Es gibt prinzipiell zwei mögliche politische Reaktionen, um die daraus resultierende Modernisierungsblockade durch die Mieter, die in deutschen Städten regelmäßig die Mehrheit stellen, zu adressieren:

1. Eine Erhöhung der Eigentümerquote würde den Widerstand gegen Aufwertungen mindern. Eigennutzer können nicht gegen ihren Willen verdrängt werden. Sie 
profitieren von der Erhöhung der Lagewertigkeit - es denn, sie planen sich in der gleichen Lage zu vergrößern.

2. Eine Deckelung der Erhöhungen von Bestandsmieten schützt Bestandsmieter. Die Mietpreisbremse macht auch eine neu zu vermietende Wohnung für untere und mittlere Einkommensgruppen vermeintlich erschwinglich. Allerdings werden Neuankömmlinge aus finanziell schwachen Milieus realistisch von der Mietpreisgrenze kaum profitieren, da Vermieter bei gleicher möglicher Miethöhe tendenziell die Bessergestellten bevorzugen werden. Und selbst für Bessergestellte ist der Schutz unsicher. Die abschließende höchstrichterliche Würdigung von Höchstmieten bei Neuverträgen fehlt, und natürlich gibt es zahlreiche Umgehungsmöglichkeiten. In München wurden beispielsweise schon 2016 rund $60 \%$ der Wohnungen nur noch möbliert angeboten (Müller 2016); hinzu kommt ein steigender Teil an Untervermietungen. Zudem implizieren Mietpreisgrenzen zahlreiche Anreizverzerrungen, die zu einer ineffizienten Verteilung von Wohnraum führen können. ${ }^{1}$ Schließlich machen Mietpreisbegrenzungen die Eigennutzung relativ weniger attraktiv, was langfristig zu einer Verstärkung der Problematik eines inhärenten Widerstandes gegen Aufwertungen führt.

Man könnte geneigt sein, 1) als die logische Konsequenz für Gebiete mit tendenziell steigender Attraktivität zu betrachten - ohne den etablierten Mieterschutz in Frage zu stellen. Zwar gibt es seit kurzem wieder ein Baukindergeld, was tendenziell die Erschwinglichkeit von Eigenheimen erleichtern soll. Allerdings scheint das Baukindergeld kaum für eine Entspannung in den Städten mit Wohnungsknappheit zu sorgen. Bis Mitte Januar 2019 wurden nur rund 12\% der Anträge für Neubauvorhaben gestellt, der große Rest betraf den Kauf von Bestandsimmobilien (N.N. 2019). Und die absolut und relativ meisten der 56.435 Anträge kommen aus Flächenländern (Nordrhein-Westfalen (12.606), Baden-Württemberg (7562), Niedersachsen (7099) und Bayern (6941)). In Berlin (861), Hamburg (589) und Bremen (492) war die Zahl vernachlässigbar.

Stattdessen hat sich die Politik stark auf 2) fokussiert. Zusätzlich zur traditionellen Regulierung der Bestandsmieten und der seit 2015 geltenden Mietpreisbremse wurde das geltende Mietrecht zum 01.01.2019 noch einmal verschärft. Erlaubt ist zum Beispiel nur noch eine Modernisierungsumlage von maximal $2 € / q m$ in einem Zeitraum von sechs Jahren, sofern die Nettokaltmiete zuvor unter $7 € / q m$ lag. Energetische Sanierungen, vor kurzer Zeit noch forciert, fallen ebenfalls unter diese Restriktionen. Gerade ,linke“ Parteien haben sich diesem Ansatz verschrieben - die Förderung des Eigenheimbesitzes spielt für sie kaum eine Rolle. Im Gegenteil: In wachsenden Teilen der „links“ regierten Städte werden durch die Ausweisung von Milieuschutzgebieten Umwandlungen in Eigentumswohnungen gezielt erschwert.

Angesichts der regressiven Umverteilungswirkung steigender Mieten und dem Anspruch, zunehmende Vermögensungleichheiten zu dämpfen, ist dies eine ablehnende Haltung zu Wohneigentum , linker“ Parteien zunächst erstaunlich. Naheliegender könnte sein, dass eine auf Gleichheit in den Lebensumständen und finanziellen

\footnotetext{
1 Mietobergrenzen können zu Überkonsum von Wohnraum von Altmieten und Unterversorgung beispielsweise junger Familien mit Kindern führen, vgl. Glaeser und Luttmer (2003).
} 
Entwicklungsmöglichkeiten ausgelegte Politik eine Gleichverteilung von Grundbesitz anstrebt, etwa in der Form, dass jeder Nutzer Eigentümer seines Wohnraumes sein soll. Schließlich ist der Erwerb eines Eigenheims ein erfolgsversprechender Weg zur Vermögensakkumulation, gerade auch für einkommensschwächere Haushalte (Mathä et al. 2017).

Dieser Artikel beschreibt die unterschiedlichen Anreizwirkungen von stadtentwicklungspolitischen Maßnahmen auf Eigennutzer und Mieter (Abschn. 2). Sodann werden in Abschn. 3 die externen Nutzen und Kosten erläutert, die mit Eigennutzungen assoziiert werden. Abschn. 4 erläutert politökonomische Ansätze, welche die Positionierung der Parteien in der Frage Mieter- versus Eigennutzer-Förderung erklären. In Abschn. 5 regen wir einen Wandel weg von einer symptomatischen Behandlung der Umverteilungswirkung städtebaulicher Entwicklung (Mietpreisregulierung) hin zur Auseinandersetzung mit den Ursachen (niedrige Eigennutzerquoten insbesondere in Großstädten) an und hinterfragen die Sinnhaftigkeit des Widerstandes gegen erhöhte Eigentumsquoten.

\section{Die unterschiedlichen Interessen von Mietern und Eigentümern und die Krux der (direkten) Demokratie}

In der Regel dürften beispielsweise ein erweiterter Park oder weniger Autoabgase in den jeweiligen Straßen bei den Anwohnern Nutzen hervorrufen, welche unabhängig sind von ihrer rechtlichen Stellung als Mieter oder Eigentümer. Allerdings sind sie unterschiedlich von den mit der Veränderung der Lagewertigkeit verbundenen Veränderungen der Mieten und Immobilienpreise betroffen (zur formalen Analyse vgl. Anhang).

In einem vollkommenen Markt (ohne Mietpreisgrenzen usw.) und bei homogenen Präferenzen werden für Mieter die erhöhten Nutzen aus der jeweiligen lokalen Lagewertigkeit vollständig über die proportional erhöhten Mieten ausgeglichen. In der Regel werden Mieter, die über ein begrenztes Einkommen verfügen, die von ihnen nachgefragte Menge an Wohnraum verringern (müssen), es sei denn sie wären bereit an anderen Gütern zu sparen. Zwar haben sie auch die Möglichkeit, in eine andere, billigere Gegend zu ziehen. Allerdings ist dort die Lagewertigkeit entsprechend geringer; im Gleichgewicht können die Mieter einer bestimmten Gegend ihren Nutzen nicht durch den Wechsel in einen anderen Stadtteil verbessern. Die vollständige Kompensation der erhöhten Lagewertigkeit durch entsprechend erhöhte Mieten macht Mieter gegenüber Veränderungen der Lagewertigkeit ihrer Umgebung indifferent.

Wenn die Präferenzen der Menschen für verschiedene Lagewertigkeiten unterschiedlich sind, kann sich das Kalkül der Mieter so ändern, dass sie gegen die Aufwertung ihrer Gegend sind: Sollte die gestiegene lokale Lagewertigkeit neue Mieter anziehen, die höhere Präferenzen für den gestiegenen Lagewert haben als die bisherigen Mieter, wird der durch die höhere Marktmiete ausgelöste Rückgang des Wohnkonsums den Lagewertigkeits-Effekt übersteigen. Diese Präferenzunterschiede werden die Regel sein; die Mieter hatten sich in der Regel bei Vertragsschluss für ihren präferierten lokalen Mix aus Lagewertigkeit und Miete entschieden. Aufgrund 
von Mobilitätskosten (z.B. Umzugskosten und Verlust von Sozialkapital) wird der Mieter auch nicht in der Lage sein, das bisherige Versorgungsniveau wiederherzustellen, indem er in ein anderes Viertel mit dem bevorzugten Mix aus Miete und Lagewertigkeit wechselt. Der negative Effekt der Mietsteigerungen reduziert seinen Gesamtnutzen trotz erhöhter Lagewertigkeit. Eine Opposition der Mieter gegen „positive“ lokale Lagewert-Veränderungen ist rational.

Verhindern Regulierungen von Mieten bzw. Mietanpassungen eine Anpassung der Bestandsmieten an die erhöhte Marktmiete, wird die Erhöhung der Mieten die gestiegene Lagewertigkeit nicht ausgleichen. In der Folge steigt der Nutzen der Alt-Mieter; die Verringerung des Konsums von Wohnraum und/oder anderer Güter fällt (geringer) aus; zusammen mit der gestiegenen Nachfrage der Mitinteressenten für die Lage ergibt sich ein durch eine Überschussnachfrage gekennzeichnetes Ungleichgewicht.

Zusammenfassend: im Vergleich zum Benchmark-Szenario aus freien Märkten und homogenen Präferenzen verringern heterogene Präferenzen die Wahrscheinlichkeit, dass Mieter positive Veränderungen der Lagewertigkeit unterstützten, während Begrenzungen von Mietsteigerungen die Wahrscheinlichkeit erhöhen.

Bei Eigentümern sieht das Kalkül anders aus: Sie erzielen zwar gleichartige zusätzliche Nutzen wie Mieter aus einem erhöhten Lagewert. Steigende Mieten treffen sie jedoch nicht. Dies kann auch gelten, wenn sie nicht selber in ihrer Wohnung leben, sondern diese vermieten und stattdessen in der gleichen Gegend eine gleichartige Wohnung mieten. Eigentümer sind gegen Mieterhöhungen abgesichert. Wenn ein Eigentümer als Reaktion auf eine Erhöhung der Lagewertigkeit beschließt, in eine Nachbarschaft mit der anfänglichen Lagewertigkeit und dem entsprechenden Mietniveau umzuziehen, realisiert er keinen Lagewert-Effekt und keine Auswirkungen auf die (implizite) gezahlte Miete, aber einen Vorteil aus der erhöhten Marktmiete aus der vermieteten Immobilie. Auch wegziehende Eigentümer, die ihre Immobilie selbst bewohnten, erzielen bei Lagewert-Erhöhungen einen Netto-Nutzenzuwachs, zumindest wenn ihre Mobilitätskosten hinreichend gering sind.

Dies gilt bereits bei homogenen Präferenzen. Sollte die gestiegene lokale Lagewertigkeit neue Mieter anziehen, die höhere Präferenzen für den gestiegenen Lagewert haben als der Eigentümer, so wird die lokale Mietsteigerung aufgrund der Lagewert-Änderung größer ausfallen als die Bewertung der Lagewertigkeit durch den Eigentümer. In diesem Fall profitiert der Eigentümer über den reinen Lagewert-Effekt, den er beim Bewohnen der Immobilie selbst erzielen würde, hinaus. Um einen ähnlichen Vermögensgewinn zu erzielen, kann der Eigennutzer seine Immobilie verkaufen und eine Immobilie in einer der ursprünglichen Lagewertigkeit entsprechenden Gegend erwerben.

Eigentümer dürften daher Initiativen, von denen erwartet wird, dass sie die Lagewertigkeit ihrer Nachbarschaft verbessern (verschlechtert), stärker unterstützen (ablehnen) als Mieter. Diese Schlussfolgerung steht im Einklang mit der sogenannten Homevoter-Hypothese (Fischel 2001), wonach Eigentümer, deren Immobilie in der Regel das größte Asset in ihrem Portfolio ist, dazu neigen, Maßnahmen zuzustimmen (abzulehnen), welche die Lagewertigkeit ihrer Immobilie erhöhen (verringern). Dies gilt, selbst wenn sie von der Maßnahme nicht direkt profitieren; so stimmen Kinderlose für eine neue Schule, wenn sie sich hierdurch eine Verbesserung der 
Lagewertigkeit versprechen (Hilber und Mayer 2009). Folgerichtig werden Eigentümer Maßnahmen tendenziell ablehnen, welche lokal negative externe Effekte für ihre Immobilie mit sich bringen, selbst wenn sie diese im Prinzip für vorteilhaft für ihre Stadt erachten. Dieses Not-in-my-Backyard-Verhalten (NIMBY) wurde mittlerweile vielfach empirisch bestätigt. Für Deutschland liegen entsprechende empirische Untersuchungen an den Beispielen der Münchener Allianz-Arena, des Berliner Flughafens und des Stuttgarter Bahnprojektes S21 vor (Ahlfeldt et al. 2014, 2019; Ahlfeldt und Maennig 2015). Entsprechend der obigen Argumentation ist dieses Verhalten für Mieter weniger ausgeprägt, da diese durch einen geringeren erwarteten Mietanstieg entschädigt werden. Ist eine Aufwertung im eigenen Viertel schon weit fortgeschritten (Stichwort Gentrifizierung) kann sich das Verhalten sogar umkehren; langjährige Bestandsmieter sind bereit Verschlechterungen im Wohnumfeld hinzunehmen, wenn sich dadurch die Attraktivität für Zuziehende verringert.

Werden Maßnahmen zur Stadtentwicklung in Volksabstimmungen oder auf anderweitig partizipatorischem Wege entschieden, so kann sich daraus eine „Krux der Direkten Demokratie“ ergeben: In Örtlichkeiten mit Mehrheiten der Mieter werden Stadtentwicklungs-Maßnahmen, welche die Lagewertigkeit (insbesondere aus Sicht potentieller Neuankömmlinge) erhöhen, schwer durchsetzbar sein. ${ }^{2}$ In Orten mit Mehrheiten der Eigentümer sind sie hingegen leichter durchsetzbar. Es dürfte aber letztlich nur dem Leitbild einer kleinen Zahl von Menschen entsprechen, dass die öffentlichen Gelder zur Stadtentwicklung vornehmlich in den Gebieten mit Eigentümer-Mehrheiten eingesetzt werden.

Die „Krux der Direkten Demokratie“ kann in ähnlicher Form auch in einer repräsentativen Demokratie gelten: Um Wählerstimmen zu maximieren, passen die Parteien ihre Wahlprogramme entsprechend an. Im Endeffekt wird aus der Krux der Direkten Demokratie die „List der Demokratie“: Selbst wenn Politiker und ihre Parteien eigentlich etwas anderes wollen, kommen sie letztlich nicht umhin sich den Präferenzen ihrer potentiellen Wähler unterzuordnen.

Es verwundert deshalb nicht, dass insbesondere in städtischen Wahlgebieten, in denen in Deutschland regelmäßig die Mieter die Mehrheiten stellen, der Begriff der „Stadtentwicklung“ zunehmend gemieden wird. Schließlich bedeutet eine Stadtentwicklung, welche erfolgreich zu einer Erhöhung der Wohn- und Aufenthaltsqualität führt, eine Bedrohung für die Bestandsmieter. In Berlin Prenzlauer Berg wurden Anfang der 90er Jahre fünf Sanierungsgebiete mit über 30.000 Wohnungen bestimmt. Nachdem viele Millionen in die Sanierungsgebiete geflossen waren, hatte sich bei deren Aufhebung nicht nur der Zustand der Gebäude deutlich verbessert (Ahlfeldt et al. 2017, 2018), sondern auch die Bevölkerungsstruktur vollkommen geändert (Büro für Stadtplanung, -forschung und -erneuerung 2008). Die zuvor gemischte Bevölkerungsstruktur wurde durch eine relativ homogene, aus dem Westen Deutschlands zugezogene Mittelschicht ersetzt. In einigen aufgehobenen Sanierungsgebieten wie dem Gebiet „Winsstraße“ betrug bereits 2007 der Anteil der Studenten und

\footnotetext{
2 Zum Zusammenhang zwischen der lokalen Ausstattung mit Infrastruktur, der Attraktivität des Standortes und der lokalen Wohnraum-Nachfrage sowie den Mieten vgl. Glaeser (2008). Für eine skeptisches Sicht zu den Absichten und Fähigkeiten von Stadtentwicklungsbehörden, tatsächlich die Lagewertigkeit zu erhöhen, vgl. Ahlfeldt et al. (2017, 2018).
} 
Hochschulabsolventen fast $77 \%$, verglichen mit 17,5\% in 1992. Die (ehemaligen) Sanierungsgebiete haben sich von den ärmsten zu den einkommensstärksten Gebieten Berlins entwickelt. Gemessen an dem Ziel bauliche Missstände zu beheben, war die Politik ein Erfolg; gemessen an dem zuvor explizit definierten Ziel die Sozialstruktur zu erhalten (Holm 2011), ein Fehlschlag.

Stattdessen sprechen die an Mietermilieus orientierten Parteien vorzugsweise von „Sozialer Stadterneuerung“. Der Gedanke ist, dass Wählern, die nach obigem Kalkül aus ,üblichen“, aufwertenden Stadtentwicklungsmaßnahmen Netto-Nachteile erleiden würden, eine Kompensation angeboten wird. Die Kompensation kann in Form des zusätzlichen Baus ,bezahlbaren Wohnraumes“, oder der zusätzlichen Bereitstellung von anderen sozialen Fazilitäten (z. B. Begegnungsstätten, Kindergärten, Schulen) geschehen. Das Spannungsfeld in dem die „Soziale Stadtentwicklung“ operiert, zeigt sich aber anschaulich daran, dass auch als „Kompensation“ gedachte neue Kindergärten und Schulen ein Quartier attraktiver machen, was langfristig zu Aufwertung und Verdrängung führen kann. Eine andere Interpretation ,Sozialer Stadtentwicklung“ ist es, aufwertenden Maßnahmen andere, tendenziell abwertende Maßnahmen entgegengenzusetzen, sodass eine monetäre Aufwertung per Saldo nicht stattfindet. Solche Gegenmaßnahmen können realisiert werden, indem in aufwertungsbedrohen Quartieren Einrichtungen mit lokal negativen externen Effekten angesiedelt werden. Dazu kann Sozialer Wohnungsbau gehören, dem - sei es aufgrund der üblichen Architektur, sei es aufgrund der typischen Bewohner - zumindest in Gebieten mit höherem Einkommen - ein negativer externer Effekt nachgewiesen wird (Diamond und McQuade 2019). Auch Drogentherapiestätten, Obdachloseneinrichtungen oder einfach nur die Erlangung von Belegungsrechten für weniger privilegierte Familien ${ }^{3}$ kommen in Frage. Bei solch „Sozialer Stadterneuerung“ sind Widerstände aus Mieterkreisen nicht oder zumindest nur eingeschränkt zu erwarten. Aus wohlfahrtsökonomischer Perspektive unter Berücksichtigung aller externen Kosten und Nutzen wird eine räumliche Allokation von Einrichtungen mit gesellschaftlich positiven, aber lokal negativen Wirkungen auf Grundlage der Eigennutzerquote kaum effizient sein. Dementsprechend wirkt dieser Ansatz als politisches Leitbild eher wie eine Verlegenheitslösung.

\section{Externe Kosten und Nutzen von eigengenutztem Wohnraum}

Wie geschildert setzt die Politik in deutschen Großstädten zur Abmilderung des Verteilungskonflikts, der sich aus städtebaulicher Aufwertung ergibt, vorzugsweise auf Mieterschutz. Umwandlungen, welche eine Erhöhung der Wohneigentumsquote nach sich ziehen würden, werden tendenziell kritisch gesehen und zum Beispiel in einigen Milieuschutzgebieten gezielt verhindert. Grundsätzlich lässt sich dieser

\footnotetext{
3 Neben (ehemaligen) Sanierungsgebieten, in denen die Preise gestiegen sind (insbesondere die oben genannten in Prenzlauer Berg) gibt es auch einige, in denen die Preise gefallen sind, z. B. im Gebiet „BeusselKiez“. Dort hatte die Sanierungsverwaltungsstelle die Sanierungsgelder im Tausch gegen Belegungsrechte vergeben, welche für vorzugsweise für Großfamilien mit Migrationshintergrund vergeben wurden, vgl. Ahlfeldt et al. (2017, 2018).
} 
Ansatz aus ökonomischer Sicht mit sozialen Kosten, die mit einer höheren Wohneigentumsquote einhergehen würden, begründen.

Gehen wir, vor einer Diskussion spezifischer externer Kosten und Nutzen, zunächst von einem idealtypischen Markt aus. In einem gut geordneten Markt werden die mündigen, rationalen Individuen auf der Anbieter- und Nachfrageseite ihr privates Kosten-Nutzen-Kakül beispielsweise auf der Grundlage der Miet-, Preisund Zinsdaten und deren erwartete Entwicklung in Kauf- oder Mietentscheidungen umsetzen. ${ }^{4}$ Nur solange die privaten (Grenz-)Nutzen des Eigentumserwerbs, unter Relation zu den Opportunitätsnutzen bei Miete, die (Grenz-)Kosten der Wirtschaftssubjekte überschreiten, kommt es zu zusätzlichen Käufen und somit einer Erhöhung der Eigennutzerquote. Da die Entscheidungen einzelner Wirtschaftssubjekte keine Wirkung auf Dritte haben, stellt sich eine gesellschaftlich optimale Eigennutzerquote ein. Im Umkehrschluss ergibt sich, dass die Aggregation der individuellen Entscheidungen nicht zu einem sozialen Optimum führt, wenn zu den privaten (Grenz-)Nutzen und (Grenz-)Kosten weitere externe Kosten und Nutzen hinzukommen.

Die in der Literatur am umfangreichsten diskutierten sozialen Kosten einer hohen Wohneigentumsquote sind in einer vermuteten verringerten räumlichen Mobilität von Eigennutzern begründet. Gemäß der sogenannten Oswald-Hypothese führt die verringerte Mobilität bei strukturellen oder konjunkturellen Schocks auf dem Arbeitsmarkt zu Anpassungsfriktionen und erhöhter Arbeitslosigkeit. Oswald (1996, 1997) zeigte in einer inter- und intranationalen Analyse verschiedener EU- und USA-Staaten auf, dass eine Erhöhung der Eigennutzerquoten um 10 Prozentpunkte zu einer Erhöhung der Arbeitslosenrate um zwei Prozentpunkte führt. Allerdings vernachlässigte er wichtige Determinanten der Arbeitslosigkeit, wie zum Beispiel die Verbreitung von Gewerkschaften. Dies holten Nickell und Layard (1999) in einer Analyse von 20 OECD Staaten nach und kamen dabei zu einem etwas verminderten Effekt von 1,5 Prozentpunkten. Green und Hendershott (2001) zeigen, dass sich unter Berücksichtigung eines ,selection bias“ unter anderem für unterschiedliche sozio-ökonomische Eigenschaften von Mietern und Eigennutzern der Effekt auf nur noch 1/8 des ursprünglichen Oswald-Effektes reduziert. Lerbs (2011) kommt in einer Untersuchung für Deutschland zu dem Schluss, dass der Zusammenhang allenfalls geringfügig ist. Nach Bracke et al. (2018) führen höhere Eigennutzerquoten ferner zu einer verringerten unternehmerischen Tätigkeit, weil die Hypothekenbelastung Zugang zu Fremdkapital erschwert und es somit schwieriger wird, ein Unternehmen zu gründen.

Diesen externen Kosten stehen jedoch externe Nutzen höherer Eigennutzerquoten gegenüber. So argumentieren Henderson und Ioannides (1983) in einem theoretischen Modell, dass eigengenutzte Immobilien besser instandgehalten werden. Mayer (1981), Galster (1983) sowie Shilling et al. (1991) bestätigen einen solchen Effekt empirisch für verschiedene Regionen in den USA. Rohe und Stewart (1996) zeigen,

\footnotetext{
4 Vgl. für Deutschland Voigtländer (2009) sowie Lerbs und Oberst (2014). Zum möglichen Einfluss steuerlicher Regeln auf das Kalkül vgl. Expertenkommission Wohnungspolitik (1995), S. 300-324. Eigennutzer in Deutschland, insbesondere in unteren Einkommensgruppen, sind zufriedener mit ihrem Leben als Mieter (Zumbro 2014); wenn dieser Effekt antizipiert wird, mag er ebenfalls zu den Determinanten einer Kaufentscheidung gehören.
} 


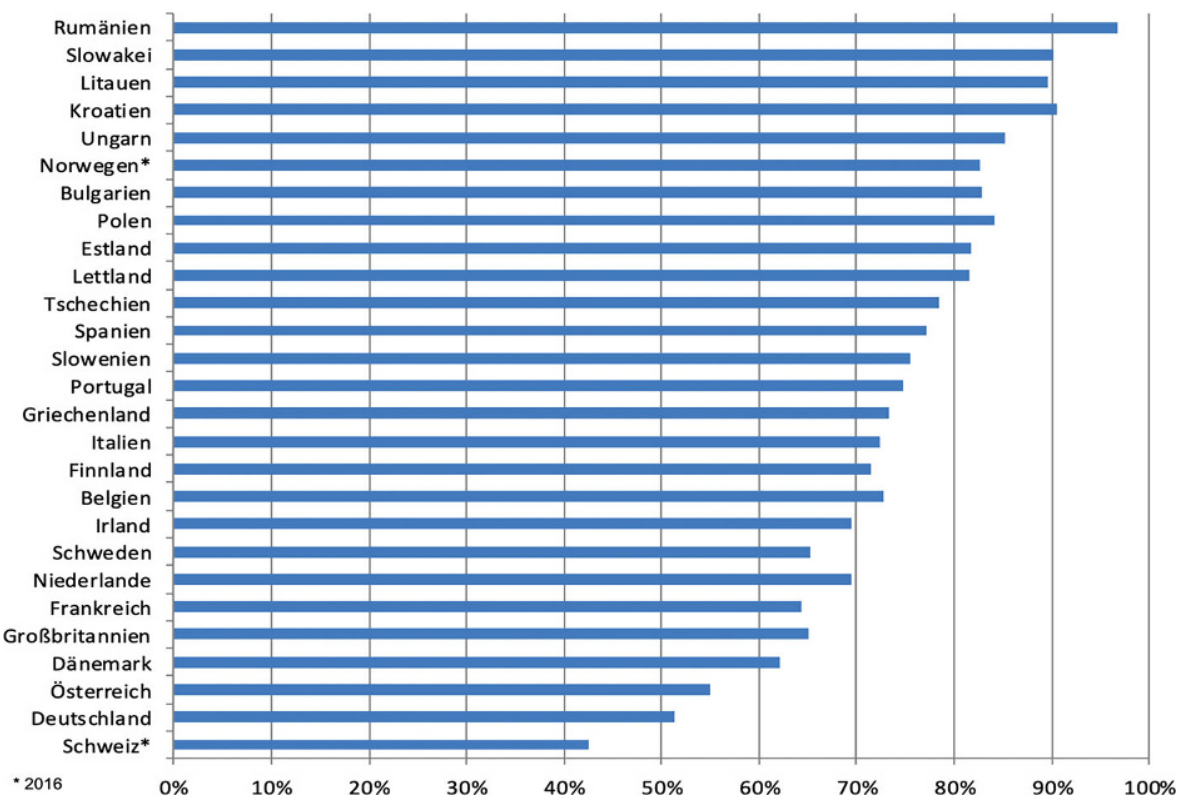

Abb. 1 Internationaler Vergleich der Eigennutzerquoten, 2017. Datenquelle: Eurostat (2018)

dass eine größere Eigennutzerquote den durchschnittlichen Wert der örtlichen Immobilien erhöht; Gatzlaff et al. (1998) berechnen, dass eigengenutzte Immobilien sich pro Jahr um 0,16 Prozentpunkte besser im Wert entwickeln als vermietete.

Eigennutzer haben darüber hinaus ein größeres Interesse daran, dass ihre örtliche Kommune gut verwaltet wird und setzen sich entsprechend mehr für die kommunalen Belange ein, oder - in der Sprache der Ökonomen: - bauen mehr Sozialkapital auf. Rossi und Weber (1996) sowie Alford und Scoble (1968) zeigen für die USA, dass Eigennutzer sich mit größerer Wahrscheinlichkeit für öffentliche Belange interessieren und auch unter Berücksichtigung ihres sozioökonomischen Status eher politisch engagiert sind. Di Pasquale und Glaeser (1999) zeigen anhand von Daten für die USA und Deutschland ebenfalls, dass Eigennutzer die „besseren Bürger“ sind. Ihre Ergebnisse bestätigen, dass Eigennutzer sich häufiger sozial und politisch engagieren, ihre Wohnungen besser instandhalten, und zudem häufiger religiöse Veranstaltungen besuchen. Blum und Kingston (1984) zeigen, dass Eigennutzer stärker ehrenamtlich engagiert sind; Dietz und Haurin (2003) heben hervor, dass sie sich stärker lokalpolitisch engagieren. ${ }^{5}$ Dieser offensichtlich verstärkte Anreiz zur Bildung von Sozialkapital ist rational; schließlich können Eigennutzer davon ausgehen, dass ein verbessertes, lokal gebundenes Sozialverhalten den Wert ihrer Immobilien tendenziell erhöht.

\footnotetext{
5 Engelhard et al. (2010) können solche Effekte für eine relativ kleine Stichprobe von Eigentümern mit niedrigem Einkommen in Tusla, Oklahoma, USA nicht nachweisen. Kortelainen und Sarimaa (2015) melden für Helsinki ebenfalls Zweifel an, da dort erhöhte Eigennutzerraten nicht mit erhöhten Immobilienpreisen einhergehen - was der Hypothese eines erhöhen Sozialkapitals widerspricht.
} 

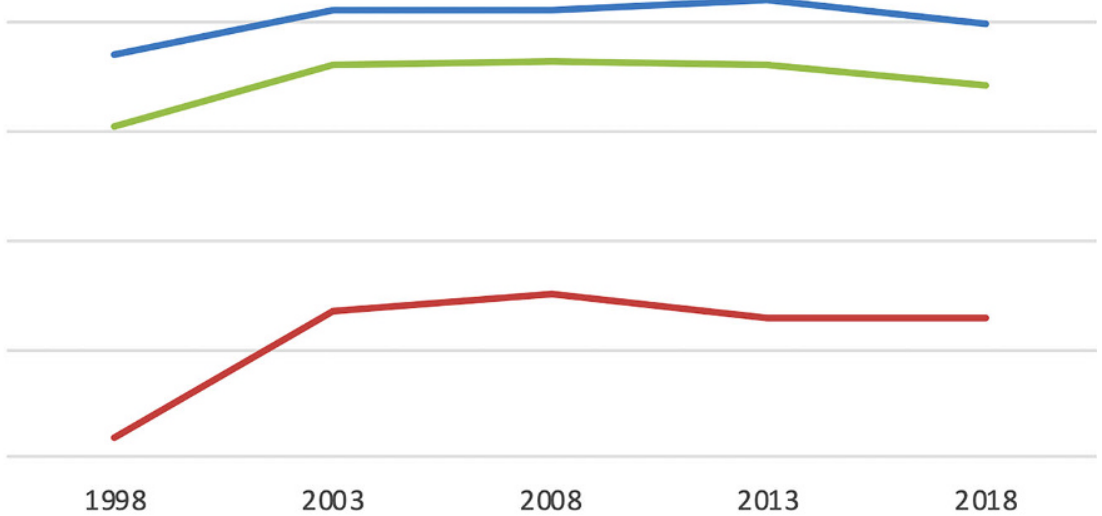

Früheres Bundesgebiet* — Neue Länder und Berlin* Deutschland

Abb. 2 Haushalte im Wohneigentum, 1998-2018. Datenquelle: Eurostat (2018)

Green und White (1997) demonstrieren mit Daten für die USA, dass Kinder von Eigennutzern mit größerer Wahrscheinlichkeit einen Schulabschluss erlangen wohlgemerkt unter Berücksichtigung einer Reihe von anderen Einflussfaktoren und nach Berücksichtigung eines möglichen ,selection bias“ aufgrund unterschiedlicher sozio-demographischer Eigenschaften von Mietern und Eigentümern. Haurin et al. (2002) zeigen mit Daten für die USA, dass die mit der Eigennutzung einhergehende höhere Qualität der Wohnumgebung zu einer um sieben bzw. neun Prozent erhöhten Lese- und Rechenleistungen der Kinder von Eigennutzern führt.

Es ist anzunehmen, dass die Höhe der externen Kosten und Nutzen einer höheren Eigennutzerquote von den Sozial- und Bildungssystemen, Mobilitätsanforderungen, aber auch von den Mobilitätskosten beispielsweise in Form von Transaktionskosten von Immobilien (Grunderwerbssteuern, Maklergebühren etc.), der Alters- und Beschäftigungsstruktur, und der Dichte der gebauten Umwelt abhängen. ${ }^{6}$

\footnotetext{
${ }^{6}$ Unabhängig sind die lokalen optimalen Eigennutzerquoten jedoch von den externen pekuniären Effekten, welche beispielsweise bei Umwandlung von Miet- in Eigentumswohnungen entstehen können. Wenn sich in einer Gesellschaft mehr Menschen für Eigentum statt Miete entscheiden, werden zunächst die Preise auf dem Eigentumsmarkt steigen. Diese Preissteigerungen werden benötigt, damit die angebotenen Mengen an Eigentumswohnungen steigen. Das zwecks Speisung der erhöhten Angebotsmengen auf dem Eigentumsmarkt reduzierte Angebot an Mietwohnungen dürfte zunächst nicht zu Mietsteigerungen führen, da die nachgefragte Mietmenge in den Eigentumsmarkt umgeschichtet wurde. Abweichungen können sich ergeben, wenn die Quadratmeter-Pro-Kopf-Nachfrage auf Eigentumsmärkten höher ist als auf Mietmärkten. Dann würden die steigenden Mieten aber letztlich nur eine erhöhte Gesamtnachfrage nach Wohnraum signalisieren. Genau dieses Signal wird benötigt, damit die angebotenen Mengen auf dem Mietmarkt wieder steigen. Übrigens: Bestandseigentümer mögen die Umwandlung von Miet- in Eigentumswohnungen in der Regel nicht. Der Wertzuwachs ihrer Immobilien bei steigender Präferenz für Eigennutzung ist gröBer, wenn das Angebot nicht ausgedehnt wird. Zum möglichen Zusammenhang zwischen wachsenden Eigennutzerquoten und wachsenden Widerstand gegen städtisches Wachstum vgl. Ortalo-Magne und Prat (2014). Grundsätzlich sind pekuniäre Effekte aus volkswirtschaftlicher Sicht kein Grund für wohnungspolitisches Eingreifen: Im Gegenteil, bei Eingriffen würden die veränderten gesellschaftlichen Knappheiten
} 
Auch der bereits erreichte Grad an Eigennutzerquoten spielt eine Rolle: bei hohen Quoten dürften der (Grenz-)Nutzen einer weiteren Erhöhung gering, die (Grenz-)Kosten hingegen höher sein. Die deutsche Eigennutzerquote ist eine der geringsten in den entwickelten Ländern (Abb. 1), wenngleich sie in den letzten Jahren auf nunmehr rund $50 \%$ gestiegen ist (Abb. 2) und ein weiteres Wachsen aufgrund der unvollständigen Anpassungsprozesse in den Neuen Ländern zu erwarten ist. Allerdings sind die Eigennutzerquoten in den Agglomerationszentren deutlich geringer (z. B. Berlin 18,4\%, Hamburg 29,8\%). In innerstädtischen Bezirken wie Berlin Kreuzberg, wo sich die Konflikte um städtebauliche Aufwertung zuspitzen, liegt die Quote teilweise unter $5 \%$ (Ahlfeldt und Maennig 2015).

Zusammenfassend bleibt festzuhalten, dass die Bestimmung der optimalen Eigennutzerquoten schwierig ist, sofern der Saldo der genannten externen Kosten und Nutzen im Vergleich zu den privaten Kosten und Nutzen eine wesentliche Größe erreicht. Nur für den Fall, dass die externen Kosten die externen Nutzen übersteigen und der Saldo im Vergleich zu den privaten Nutzen und Kosten wesentlich ist, wäre eine Eigennutzerquote, welche sich aufgrund von Marktkräften ergibt, aus gesellschaftlicher Sicht zu hoch. Ein solcher Nachweis liegt jedoch nicht vor. Für die deutschen Agglomerationszentren ist es besonders unwahrscheinlich, dass die externen (Grenz-)Kosten der Eigennutzung die externen (Grenz-)Nutzen überschreiten. Insofern besteht derzeit in den deutschen Großstädten keine Legitimation, der politischen Entscheidungsfindung ein Primat gegenüber dem methodologischen Individualismus einzuräumen und steigende Eigennutzerquoten zu behindern.

\section{Erklärungsansätze hesitanter Eigenheim-Politik}

Es liegt keine empirische Evidenz dafür vor, dass steigende Eigennutzerquoten in den deutschen Agglomerationszentren regional- bzw. volkswirtschaftlich unvorteilhaft sind. Im Gegenteil: die oben genannten Kosten erscheinen in Agglomerationszentren mit ihren typischer Weise geringen Eigennutzerquoten relativ klein im Verhältnis zu den Nutzen, insbesondere, wenn die in Abschn. 2 genannten, mit hohen Mieterquoten verbundenen verteilungspolitischen Implikationen der Stadtentwicklung betrachtet werden.

Insofern ist es zunächst erstaunlich, dass die Parteien in Deutschland im Vergleich zu anderen westlichen Industrieländern eine nur begrenzte Präferenz für die Erhöhung der Eigennutzerquoten haben, sondern dazu neigen, über Mietsteigerungsbegrenzungen das Wohneigentum relativ unattraktiv zu machen. Kohl (2018) wertet 1809 Wahlprogramme in 19 Ländern seit 1945 aus und kommt unter anderem zu dem Ergebnis, dass in den 69 Wahlprogrammen deutscher Parteien seit 1949 in rund $62 \%$ der Fälle Positionen pro Eigennutzung enthalten sind (einer der international geringsten Quoten), in $61 \%$ der Fälle Positionen pro Mieter (eine der höchsten Quoten), und in $42 \%$ Position zu Mietbegrenzungen (der mit Abstand höchste internationale Wert).

kaschiert; der Anpassungsprozess an die gesellschaftlichen Präferenzen würde verunmöglicht oder verzögert. 
Dabei sind in den internationalen Parteiprogrammen zu $94 \%$ in den konservativen Parteien Positionen pro Eigennutzung enthalten; bei den sozialdemokratischen zu $67 \%$, bei den sozialistischen Parteien zu $38 \%$ und bei den grünen Parteien zu $18 \%$. Mieterunterstützung wird von praktisch allen sozialistischen Parteien versprochen und in $84 \%$ der Programme der grünen Parteien, $77 \%$ der sozialdemokratischen Parteien, und $49 \%$ der christdemokratischen Parteien. Hingegen beinhalten nur rd. $25 \%$ der konservativen und liberalen Parteiprogramme Positionen pro Mieter.

Vor dem Hintergrund, dass die egalitäre Verteilung des Grundvermögens im Einklang mit dem Ideal sozialer Gerechtigkeit steht, erscheint die reservierte Haltung der nicht-konservativen Parteien pro Eigennutzung erst einmal nicht unmittelbar einleuchtend. Häufig wird das Argument angeführt, dass Immobilieneigentum für sozial Schwächere nicht zu realisieren sei und man sich deshalb um die weniger privilegierten Mieter besonders stark kümmern müsse. Dem ist kaum zu widersprechen und dennoch, oder gerade deswegen, bleibt die Frage im Raum warum eine soziale Politik nicht genau hier den Hebel ansetzten sollte, um das Immobilieneigentum für breitere Bevölkerungsgruppen erreichbar zu machen.

Erklären ließe sich die Fokussierung auf Mieterschutz (und implizite Anreize für eine niedrige Eigentumsquote) durch eine parteipolitische Basis, die im hier und jetzt überwiegend zur Miete wohnt und daher kurzfristig primär an Mieterschutz interessiert ist. Allerdings lag der durchschnittliche Anteil der SPD- und Juso-Mitglieder mit Immobilienbesitz zwischen 1980 und 1992 bei rund 56\%, und damit höher als im bundesdeutschen Durchschnitt. Zum Vergleich: Bei den Mitgliedern von CSU und CDU lag die Quote der Immobilieneigentümer noch 1994 bei $51 \%$, also deutlich niedriger, und stieg erst bis 2014 auf $65 \%$ (Kohl 2018). ${ }^{7}$

Wenn auch die Mitgliederzusammensetzung keine hinreichende Erklärung für die zögerliche Haltung liefert, so kann das Interesse potentieller Wähler aus politökonomischer Sicht natürlich dennoch herangezogen werden, sofern diese im Durchschnitt eine geringere Eigennutzerquote aufweisen - Stichwort „List der Demokratie“. Allerdings sind $49 \%$ der SPD Wähler bereits Immobilieneigentümer (Kohl 2018). Zudem würden rund $84 \%$ der Deutschen ,eindeutig“ $(63,3 \%)$ oder ,eher“ (20\%) eine eigene Wohnung gegenüber einer Mietwohnung bevorzugen (Kaiser 2018). Es fällt daher schwer zu glauben, dass die Wählerbasis linker Parteien politischen Maßnahmen, die den Erwerb von Eigentum erleichtern würden, nicht aufgeschlossen gegenüberstehen würde. Zumal sich Programme, die Bestandsmieter vor exzessiven Mieterhöhungen schützen und Programme, die den Erwerb von Wohneigentum fördern, nicht ausschließen. Somit bleibt als politökonomischer Erklärungsansatz für das Paradoxon linker Wohnungsmarktpolitik noch, dass eine Reihe jüngerer Studien zeigen, dass Immobilieneigentümer mit größerer Wahrscheinlichkeit an Wahlen teilnehmen (André et al. 2017), und dann für konservative Parteien stimmen (Ansell 2014).

\footnotetext{
7 Die genannten Quoten der Immobilieneigentümer mögen allerdings aufgrund des Survey-Designs leicht nach oben verzerrt sein.
} 


\section{Schlussfolgerung und Empfehlungen für die Politik}

Der Wunsch nach (mehr) Wohneigentum ist in Deutschland ungebrochen. Offensichtlich kommen die Individuen in ihren (impliziten oder expliziten) Berechnungen zu dem Ergebnis, dass ihre zusätzlichen Nutzen aus Wohneigentum (gegenüber dem Wohnen zur Miete) größer sind als die zusätzlichen Kosten. Allerdings gelingt es nicht allen Haushalten, diesen Wunsch in die Tat umzusetzen. Der Anteil der Haushalte, die Wohneigentum anstreben, übersteigt noch immer den Anteil der Haushalte, die ihre Wohnung besitzen.

Die theoretische und empirische Literatur zu den externen Nutzen und Kosten der Eigennutzung liefert keine überzeugenden Argumente, welche ein politisches Gegensteuern gegen steigende Eigennutzerquoten rechtfertigen; dies gilt insbesondere in den deutschen Agglomerationszentren mit ihren typischer Weise geringen Eigennutzerquoten. Neben erhöhten Investitionen in Sozialkapital führen wir im vorliegenden Beitrag als weiteren externen Nutzen der Eigennutzung an, dass die regressiven Verteilungswirkungen einer auf Verbesserung von Lebensumständen bedachten Stadtentwicklungspolitik verringert werden: Während Eigentümer von Entwicklungsmaßnahmen in ihrer Umgebung meist gewinnen, verlieren Mieter in der Regel. Der zunehmende Widerstand von Mietern gegen Stadtentwicklungsmaßnahmen ist rational.

Die nicht-konservativen Parteien unterstützen in Deutschland jedoch kaum die Wohneigentumsbildung. Priorität haben der Mieterschutz und die Investitionen in Sozialen Wohnungsbau bzw. Bezahlbaren Wohnraum. Da sich diese Ansätze und eine wohlwollende Haltung zum Wohneigentum nicht ausschließen bietet sich eine politökonomische Erklärung für die reservierte Haltung an: nach der empirischen Evidenz wählen Eigennutzer in der Regel konservativer.

In Anbetracht der externen Nutzen erscheint es jedoch sowohl im Sinne der ökonomischen Effizienz als auch der sozialen Gerechtigkeit an der Zeit, den Wünschen der Bevölkerung zu folgen, und insbesondere sozial Schwächere so zu unterstützen, dass sie Eigentum erwerben können.

Als wohnungspolitische Maßnahmen zur Förderung des Wohneigentums kommen neben dem seit Mitte September 2018 beantragungsfähigen Baukindergeld in Frage:

- Ein Kreditprogramm der KfW, welches das oft fehlende Eigenkapital bereitstellt. Häufig scheitern die Vorkaufsrechte der Mieter oder sonstige Kaufwünsche nicht an der langfristigen Tragbarkeit der notwendigen Kredite, sondern am mangelnden Eigenkapital. Das „,help to buy scheme“ in Großbritannien könnte als Inspiration dienen, wobei ein solches Programm in Deutschland größere soziale Nutzen stiften dürfte als in Großbritannien, wo auf Grund der notorisch geringen Bautätigkeit (Hilber und Vermeulen 2016), die Zuwendungen größtenteils in höheren Wohnungspreisen verpuffen.

- Alternativen zur KfW wären zum einen das schweizerische Modell, wonach Vorsorgegelder bzw. angesparte Rentenansprüche eingesetzt werden dürfen. In der Schweiz machen $58 \%$ aller Immobilienkäufer davon Gebrauch, wobei sie durchschnittlich rd. 100.000 SFR verwenden. In Deutschland ist dies bislang lediglich mit Mitteln aus der Riesterrente möglich. Zum anderen könnte das fehlende Ei- 
genkapital über crowdlending bereitgestellt werden, das allerdings in Deutschland noch ein Nischendasein fristet. Der Staat könnte diese Finanzierungsform stärken, indem eine angemessenene Zahl von Start-ups in dieser Branche unterstützt würden (Voigtländer 2019).

- Eine Resozialisierung mit anschließender Veräußerung an Eigennutzer, insbesondere in den Neuen Ländern. Hier waren die Immobilien lange Zeit Gemeineigentum, wurden dann jedoch an einzelne private Investoren verkauft. In einigen osteuropäischen Ländern bekamen hingegen die Mieter ihre Wohnungen übereignet; die Chance auf entsprechend hohe Eigennutzungsraten wie in Bulgarien und $\mathrm{Ru}-$ mänien wurden in Deutschland vertan. Solange in Teilen des Beitrittsgebietes Immobilien noch günstig, insbesondere unterhalb der Baukosten, zu erwerben sind, sollte über einen staatlichen Ankauf und einen Abverkauf an sozial Schwächere zu Sonderkonditionen (z. B. Eigenkapitalhilfe, Prämien bei Freimachung von gröBeren Wohnungen in Gebieten mit Wohnungsknappheit etc.) nachgedacht werden. In anderen Gebieten, wo die Preise derzeit über den Wiederherstellungskosten liegen, sollte eine der kommenden Schwächephasen im Immobilienzyklus genutzt werden, die entsprechenden Aufkäufe vorzunehmen. Dieses sollte rechtzeitig also bald - vorbereitet werden.

Zögernden Mitgliedern in nicht-konservativen Parteien können darauf hingewiesen werden, dass die Mehrheit der sozialistischen Wähler in Frankreich (76\%), der Labour-Wähler in Großbritannien (64\%), der Demokraten in den USA (65\%) und der Sozialdemokraten in Schweden (59\%) Hauseigentümer sind, wenngleich die Angaben aufgrund der dort insgesamt höheren Eigennutzerquoten nicht direkt mit Deutschland vergleichbar sind. In Deutschland sind 49\% der SPD-Wähler Immobilieneigentümer (Kohl 2018). Gerade Parteien aus dem linken politischen Spektrum sollten sich die Partizipation breiter Bevölkerungsschichten an Grund und Boden und den impliziten Renditen - zur Agenda machen. Anstatt sich von der Sorge vor einer Erosion der Wählerbasis leiten zu lassen, bietet sich als alternative Strategie an, die Deutungshoheit über das Thema zu erlangen. Aus Sicht der nicht-konservativen Parteien dürfte wohl die berechtigte Hoffnung bestehen, dass Menschen, die erst durch aktive ,linke Politik“ den Traum vom Eigenheim realisieren konnten, als Wähler erhalten bleiben oder sogar gewonnen werden können.

\section{Anhang}

\section{Veränderungen der Lagewertigkeit und die impliziten Kalküle von Mietern und Eigentümern}

Mieter und Eigennutzer mögen bei Veränderungen der Lagewertigkeit der Umgebung ihrer Wohnungen vergleichbare direkte Nutzeffekte erwarten; allerdings sind die von ihnen erwarteten Kapitalisierungseffekte aufgrund der Veränderungen der Mieten und Immobilienpreise unterschiedlich. 


\section{Mieter}

Ein Mieter erzielt in einer bestimmten Nachbarschaft einen Nutzen aus dem Konsum von Wohndienstleistungen $H$ (housing services) und der jeweiligen lokalen Lagewertigkeit $Z$ :

$$
U^{R}=f(H(r), Z), \frac{\partial f}{\partial H}>0, \frac{\partial f}{\partial Z}>0
$$

Der Mieter gebe einen fixen Betrag $\bar{W}=r H$ für Miete und Nebenkosten aus, wobei $r$ die lokale Miethöhe bezeichnet. Wir gehen von einem fixen Betrag aus, da der Anteil, den Menschen für Wohnen ausgeben, über Bevölkerungsgruppen und Regionen hinweg tendenziell relativ konstant ist (Davis und Ortalo-Magné 2011). Die Nachfrage nach Wohnraum ist durch die Budgetrestriktion wie folgt definiert:

$$
H=\frac{\bar{W}}{r}, \frac{\partial H}{\partial r}<0
$$

Angesichts des festen Budgets reduzieren höhere Mieten indirekt den Nutzwert, indem sie den Wohnverbrauch verringern. Im Gleichgewicht sind die Mieter annahmegemäß zwischen den verschiedenen Standorten indifferent; höhere Mieten gleichen die höhere Lagewertigkeit aus.

$$
\frac{\partial f}{\partial Z}=-\frac{\partial f}{\partial H} \frac{\partial H}{\partial \widetilde{r}} \frac{d \widetilde{r}}{d Z},-\frac{\frac{\partial f}{\partial Z}}{\frac{\partial f}{\partial H} \frac{\partial H}{\partial r}}=\frac{d \widetilde{r}}{d Z}>0,
$$

wobei $d \widetilde{r} / d Z$ die Zahlungsbereitschaft für Änderungen der Lagewertigkeit darstellt, ähnlich eines impliziten (hedonischen) Preises (Rosen 1974). Im Gleichgewicht können die Mieter einer bestimmten Gegend ihren Nutzen nicht durch den Wechsel in einen anderen Stadtteil verbessern.

Der Nutzeffekt einer exogenen Veränderung der lokalen Lagewertigkeit entspricht:

$$
d U^{R}=\frac{\partial f}{\partial Z} d Z+\frac{\partial f}{\partial H(r)} \frac{\partial H}{\partial r} \frac{d r}{d Z} d Z, \frac{d U^{R}}{d Z}=\frac{\partial f}{\partial Z}+\frac{\partial f}{\partial H(r)} \frac{\partial H}{\partial r} \frac{d r}{d Z} .
$$

Eine positive Änderung der Lagewertigkeit $d Z$ beeinflusst den Nutzen des Mieters zunächst direkt über dessen Bewertung der Änderung der Lagewertigkeit ( $\partial f / \partial Z)$. Hinzu kommt der indirekte Effekt, der Kapitalisierungseffekt, wenn aus der Änderung der Lagewertigkeit eine Anpassung der Marktmiete $(d r / r Z)$ erfolgt. Ein Anstieg der Marktmiete, der aus dem Wettbewerb der Mietinteressenten für eine bestimmte Wohnlage resultiert und das räumliche Gleichgewicht wiederherstellt, zwingt den Einzelnen, den Wohnkonsum am entsprechenden Ort zu reduzieren. Mit anderen Worten: In einer Welt homogener Präferenzen werden Veränderungen der Lagewertigkeit durch entsprechende Veränderungen der Mieten ausgeglichen, also 
$d r / d Z=d \widetilde{r} / d Z$. Entsprechend ergibt sich aus (1), dass der Einzelne gegenüber Änderungen der Lagewertigkeit indifferent ist:

$$
\frac{d U^{R}}{d Z}=0
$$

Wenn jedoch die Präferenzen für verschiedene Lagewertigkeiten unterschiedlich sind, kann ein Anstieg der lokalen Lagewertigkeit neue Mieter mit höheren Präferenzen für den gestiegenen Lagewert $\partial f^{*} / \partial Z>\partial f / \partial Z$ anziehen, sodass die Anpassung der Marktmiete größer ist als die Bewertung durch die ortsansässigen, etablierten Mieter, $d r / d Z>d \widetilde{r} / d Z=-[(\partial f / \partial H)(\partial H / \partial \widetilde{r}) d \widetilde{r} / d Z]$. Für einen Mieter, der nicht umzieht, wird der durch die höhere Marktmiete ausgelöste Rückgang des Wohnkonsums den Lagewertigkeits-Effekt übersteigen. Aufgrund von Mobilitätskosten (z. B. Umzugskosten und Verlust von Sozialkapital) wird der Mieter auch nicht in der Lage sein, das bisherige Versorgungsniveau wiederherzustellen, indem er in ein anderes Viertel mit dem bevorzugten Mix aus Miete und Lagewertigkeit wechselt, folglich ist $d U^{R} / d Z<0$. Der negative Effekt der Mietsteigerungen reduziert seinen Gesamtnutzen trotz erhöhter Lagewertigkeit. Eine Opposition der Mieter gegen ,,positive“ lokale Lagewert-Veränderungen ist rational.

Umgekehrt können Regulierungen von Mieten bzw. Mietanpassungen dazu führen, dass die Erhöhung der Mieten die gestiegene Lagewertigkeit nicht ausgleicht: $\frac{d r}{d Z}<\frac{d r}{d Z}$ und $\frac{d U^{R}}{d Z}>0$. In der Folge steigt der Nutzen der Alt-Mieter; die Verringerung des Konsums von Wohnraum fällt (geringer) aus; zusammen mit der gestiegenen Nachfrage der Mitinteressenten für die Lage ergibt sich ein durch eine Überschussnachfrage gekennzeichnetes Ungleichgewicht.

Zusammenfassend: Im Vergleich zum Benchmark-Szenario aus freien Märkten und homogenen Präferenzen verringern heterogene Präferenzen die Wahrscheinlichkeit, dass Mieter positive Veränderungen der Lagewertigkeit unterstützen, während Begrenzungen von Mietsteigerungen die Wahrscheinlichkeit erhöhen.

\section{Eigentümer}

Der Nutzen von Eigentümern kann wie folgt ausgedrückt werden:

$$
U^{O}=f(H(r, \bar{r}), Z),
$$

wobei $\bar{r}$ die Nettomiete nach Steuern ist, für welche Eigentümer ihre Wohnungen potenziell vermieten können. In Ländern ohne Besteuerung von Wohneigentum (und ohne Steuerermäßigung für die Kosten des Wohneigentums, beispielsweise in Form der Anrechenbarkeit von Zinszahlungen) beträgt die Nettomiete im Gleichgewicht $\bar{r}=r$. Die Budgetbeschränkung des Eigentümers hat die Form:

$$
\bar{W}+\bar{r} \bar{H}=\bar{m}+r H,
$$

wobei $\bar{m}$ eine feste periodische Hypothekenzahlung in Höhe des für die Wohnleistungen verfügbaren Budgets $(\bar{W}=\bar{m})$ ist und $\bar{H}$ die Menge der Wohnleistungen ist. Die tatsächlich konsumierte Menge an Wohnleistungen $H$ können von den Mie- 
ten abhängen, zu denen Wohnleistungen gemietet $(r)$ oder vermietet werden können $(\bar{r})$, falls der Eigentümer beschließt, seine Wohnung nicht selbst zu nutzen. Wenn der Eigentümer in derselben Nachbarschaft (oder in seiner eigenen Immobilien) bleibt, vereinfacht sich die Budgetgleichung $\mathrm{zu} \bar{W}=r H$ und der Verbrauch von Wohnleistungen entspricht $H=\bar{H}$.

$$
H=\frac{\bar{W}-\bar{m}}{r}+\frac{\bar{r}}{r} \bar{H}, \quad \frac{\partial H}{\partial r}<0, \frac{\partial H}{\partial \bar{r}}>0
$$

An einem bestimmten Ort realisiert ein Eigentümer den gleichen Lagewert-Effekt $(Z)$ wie ein Mieter, mit $-(\partial f / \partial Z) /[(\partial f / \partial H)(\partial H / \partial \widetilde{r})]=d \widetilde{r} / d Z$. Somit gilt im Gleichgewicht:

$$
-\frac{\frac{\partial f}{\partial Z}}{\frac{\partial f}{\partial H} \frac{\partial H}{\partial p}}=\frac{d r}{d Z}=\frac{d \bar{r}}{d Z}=\frac{d \widetilde{r}}{d Z}
$$

Bei den Eigentümern ist im Gegensatz zu den Mietern der (potenzielle) Kapitalisierungseffekt, der durch eine Lagewert-Änderung $d Z$ induziert wird, ein zusammengesetzter Effekt, der sich aus einer Änderung der für Wohnleistungen gezahlten Miete und einer Änderung der Mieteinnahmen aus der Vermietung seiner Immobilie ergibt.

$$
\frac{d U^{O}}{d Z}=\frac{\partial f}{\partial Z}+\frac{\partial f}{\partial H} \frac{\partial H}{\partial r} \frac{d r}{d Z}+\frac{\partial f}{\partial H} \frac{\partial H}{\partial \bar{r}} \frac{d \bar{r}}{d Z}
$$

Wohnen die Eigentümer in ihren eigenen Immobilien, zahlen und erhalten sie keine Miete und der Nutzeffekt wird ausschließlich durch den Lagewert-Effekt bestimmt. Dasselbe gilt, wenn die Immobilie vermietet ist und die Präferenzen homogen sind $(d r / d Z=d \widetilde{r} / d Z=d \bar{r} / d Z)$. Es besteht kein Anreiz, die Nachbarschaft zu verlassen, da sich die Auswirkungen von Mietsteigerungen auf den Wohnkonsum gegenseitig aufheben, d.h. $(\partial H / \partial r(Z) / \partial H / \partial \bar{r}(Z)=1)$ :

$$
\frac{d U^{O}}{d Z}=\frac{\partial f}{\partial Z}
$$

Bei Erhöhungen der Lagewertigkeit unterscheidet sich die positive Summe von Lagewert- und Kapitalisierungseffekten eines Eigentümers somit von der eines Mieters, der keinen Nettovorteil erzielen kann (3).

Wenn ein Eigentümer als Reaktion auf eine Änderung der örtlichen Gegebenheiten beschließt, in eine Nachbarschaft mit der anfänglichen Lagewertigkeit $(Z)$ und Mietpreisen $(r)$ der ursprünglichen Nachbarschaft umzuziehen, wird er keinen Lagewert-Effekt $(d Z=0)$ und keine Auswirkungen auf die (implizite) gezahlte Miete $(d r=0)$, aber einen Vorteil aus der erhöhten Marktmiete aus der vermieteten Immobilie realisieren.

$$
\frac{d U^{O}}{d Z}=\frac{\partial f}{\partial H} \frac{\partial H}{\partial \bar{r}} \frac{d \bar{r}}{d Z}
$$


die, wie in (4) definiert, dem Lagewert-Effekt $\partial f / \partial Z$ in einer Welt mit homogenen Präferenzen entspricht, weil $\frac{\tilde{d r}}{d Z}=\frac{d \bar{r}}{d Z}$.

Sind die Präferenzen jedoch heterogen und ist die Veränderung des lokalen Mietniveaus nach der Lagewert-Änderung größer als dessen Bewertung der Lagewertigkeit $(d \bar{r} / d Z>d \widetilde{r} / d Z)$, profitiert der Eigentümer über den reinen Lagewert-Effekt hinaus. Der Effekt kann in einen Quasi-Lagewert-Effekt $\partial f / \partial Z$ zerlegt werden, der einer Mietsteigerung gleichkommt: in die Bewertung $((\partial f / \partial H)(\partial H / \partial \widetilde{r})(d \widetilde{r} / d Z))$ und in einen zusätzlichen Nutzeffekt, der aus der Zuwanderung von Mietern mit höheren Bewertungen stammt $((\partial f / \partial H)(\partial H / \partial \bar{r})((d \bar{r}-d \widetilde{r}) / d Z))$ :

$$
\frac{d U^{O}}{d Z}=\frac{\partial f}{\partial H} \frac{\partial H}{\partial \widetilde{r}} \frac{d \widetilde{r}}{d Z}+\frac{\partial f}{\partial H} \frac{\partial H}{\partial \bar{r}}\left(\frac{d \bar{r}}{d Z}-\frac{d \widetilde{r}}{d Z}\right)
$$

Der zweite Summand beschreibt einen echten Kapitalisierungsvorteil, d.h. eine Erhöhung des Nutzens, die sich aus einem Vermögenszuwachs ergibt und zusätzlich zum direkten Lagewert-Effekt eintritt.

Zusammenfassend stehen Eigentümer Lagewert-Erhöhungen positiv gegenüber. Bei homogenen Präferenzen ist dies über den Lagewert-Effekt begründet. Bei heterogenen Präferenzen, die sich dadurch auszeichnen, dass (potentielle) Zuwanderer eine stärkere Präferenz für die erhöhte Lagewertigkeit haben als die Bestandsbewohner, werden sie zusätzlich von einem Kapitalisierungsvorteil profitieren, wenn der Vermögenseffekt im Verhältnis zu den Mobilitätskosten groß ist.

Open Access Dieser Artikel wird unter der Creative Commons Namensnennung 4.0 International Lizenz (http://creativecommons.org/licenses/by/4.0/deed.de) veröffentlicht, welche die Nutzung, Vervielfältigung, Bearbeitung, Verbreitung und Wiedergabe in jeglichem Medium und Format erlaubt, sofern Sie den/die ursprünglichen Autor(en) und die Quelle ordnungsgemäß nennen, einen Link zur Creative Commons Lizenz beifügen und angeben, ob Änderungen vorgenommen wurden.

\section{Literatur}

Ahlfeldt GM, Maennig W (2015) Homevoters vs. leasevoters: a spatial analysis of airport effects. J Urban Econ 87:85-99

Ahlfeldt GM, Maennig W, Ölschläger M (2014) Measuring and quantifying lifestyles and their impact on public choices: the case of professional football in Munich. J Econ Soc Meas 39:59-86

Ahlfeldt GM, Maennig W, Richter FJ (2017) Urban renewal after the Berlin wall: a place-based policy evaluation. J Econ Geogr 17:129-156

Ahlfeldt GM, Maennig W, Richter FJ (2018) Zoning in reunified Berlin. In: Lehavi A (Hrsg) One hundred years of zoning and the future of cities. Springer, Cham, S 123-138

Ahlfeldt GM, Maennig W, Steenbeck M (2019) Direct democracy and intergenerational conflicts in ageing societies. J Reg Sci. https://doi.org/10.1111/jors.12448

Alford RR, Scoble HM (1968) Sources of local political involvement. Am Polit Sci Rev 62:1192-1206

André S, Dewilde C, Luijkx R (2017) The tenure gap in electoral participation: instrumental motivation or selection bias? Comparing homeowners and tenants across four housing regimes. Int J Comp Sociol 58:241-265

Ansell BW (2014) The political economy of ownership: housing markets and the welfare state. Am Polit Sci Rev 108:383-402

Blum TC, Kingston PW (1984) Homeownership and social attachment. Sociol Perspect 27:159-180

Bracke P, Hilber C, Silva O (2018) Mortgage debt and entrepreneurship. J Urban Econ 103:52-66 
Büro für Stadtplanung, -forschung und -erneuerung (2008) Studie zur abschließenden Überprüfung der sozialen Sanierungsziele (Bevölkerungsstruktur/Mietenentwicklung) „Sanierungsgebiet Kollwitzplatz“ im Bezirk Pankow von Berlin. Berlin

Davis M, Ortalo-Magné F (2011) Household expenditures, wages, rents. Rev Econ Dyn 14:248-261

Diamond R, McQuade TJ (2019) Who wants affordable housing in their backyard? An equilibrium analysis of low income property development. J Polit Econ. Erscheint demnächst

Dietz RD, Haurin DR (2003) The social and private micro-level consequences of homeownership. J Urban Econ 54:401-450

Dipasquale D, Glaeser EL (1999) Incentives and social capital: are homeowners better citizens? J Urban Econ 45:354-384

Engelhardt GV, Eriksen MD, Gale WG, Mils GB (2010) What are the social benefits of homeownership? Experimental evidence for low-income households. J Urban Econ 67:249-258

Expertenkommission Wohnungspolitik (1995) Wohnungspolitik auf dem Prüfstand. Mohr, Tübingen

Fischel FA (2001) The homevoter hypothesis. Harvard University Press, Cambridge, MA

Galster GC (1983) Empirical evidence on cross tenure differences in home maintenance and condition. Land Econ 59:107-113

Gatzlaff DH, Green RK, Ling DL (1998) Revisiting cross-tenure differences in housing maintenance. Land Econ $74: 328-342$

Glaeser E (2008) Cities, agglomeration and spatial equilibrium. Oxford University Press, Oxford

Glaeser EL, Luttmer EFP (2003) The misallocation of housing under rent control. Am Econ Rev 93:1027-1046

Green RK, Hendershott PH (2001) Home-ownership and the duration of unemployment: a test of the Oswald hypothesis. https://www.researchgate.net/publication/228638808_Home-ownership_and_the_ duration_of_unemployment_a_test_of_the_Oswald_hypothesis?enrichId=rgreq-8f150abc0c318c024 53ce89402ddb891-XXX\&enrichSource=Y292ZXJQYWdlOzIyODYzODgwODtBUzo5ODY5NDA 0OTMwNDU5M0AxNDAwNTQxOTAzODMw\&el=1_x_2\&_esc=publicationCoverPdf. Zugegriffen: 02.02.2019

Green RK, White MJ (1997) Measuring the benefits of homeowning: effects on children. J Urban Econ 41:441-461

Hackenbruch F, Simon E (2018) Kritik an Google-Absage: „Fatales Zeichen“. Der TAGESSPIEGEL vom 25.10.2018. https://www.tagesspiegel.de/berlin/google-campus-in-kreuzberg-kritik-an-googleabsage-fatales-zeichen/23231642.html. Zugegriffen: 02.02.2019

Haurin DR, Parcel TL, Haurin RJ (2002) Does home ownership affect child outcomes? Real Estate Econ 30:635-666

Henderson JV, Ioannides YM (1983) A model of housing tenure choice. Am Econ Rev 73:98-113

Hilber CAL, Mayer CJ (2009) Why do households without children support local public schools? J Urban Econ 65:74-90

Hilber CAL, Vermeulen W (2016) The impact of supply constraints on house prices in England. Econ J 126:358-405

Holm A (2011) Berlin: Auf dem Weg in die Zitadellenökonomie. Gentrification Blog. http:// gentrificationblog.wordpress.com/2010/08/06/berlin-auf-dem-weg-in-die-zitadellenokonomie/. Zugegriffen: 17. März 2011

Kaiser S (2018) 84 Prozent der Deutschen wollen lieber ein Eigenheim statt Miete zahlen. http:// www.spiegel.de/wirtschaft/soziales/immobilien-84-prozent-der-deutschen-wollen-ein-eigenheima-1223288.html. Zugegriffen: 23. Dez. 2018

Kohl S (2018) The political economy of homeownership: a comparative analysis of homeownership ideology through party manifestos. Socioecon Rev 14:1-26. https://doi.org/10.1093/ser/mwy030

Kortelainen M, Saarimaa T (2015) Do urban neighborhoods benefit from homeowners? Evidence from housing prices. Scand J Econ 117:28-56

Lerbs OW (2011) Is there a link between homeownership and unemployment? Evidence from German regional data. Int Econ Econ Policy 8:407-426

Lerbs OW, Oberst CA (2014) Explaining the spatial variation in homeownership rates: results for German regions. Reg Stud 48:844-865

Mathä TY, Porpiglia A, Ziegelmeyer M (2017) Household wealth in the Euro area. The importance of intergenerational transfers, homeownership and house price dynamics. J Hous Econ 35:1-12

Mayer NS (1981) Rehabilitation decisions in rental housing: an empirical analysis. J Urban Econ 10:76-94

Müller B (2016) Vermieter umgehen Preisbremse mit möblierten Wohnungen. Süddeutsche Zeitung vom 04.09.2016. https://www.sueddeutsche.de/wirtschaft/wohnungsmarkt-vermieter-umgehenpreisbremse-mit-moeblierten-wohnungen-1.3147527. Zugegriffen: 4. Febr. 2019 
N.N. (2019) Baukindergeld sorgt kaum für Neubauten. http://www.spiegel.de/wirtschaft/soziales/ wohnungsnot-baukindergeld-sorgt-kaum-fuer-neubauten-a-1248958.html. Zugegriffen: 31. Jan. 2019

Nickell S, Layard R (1999) Labor market institutions and economic performance. In: Ashenfelter O, Card D (Hrsg) Handbook of labor economics, 3. Aufl. Elsevier, Amsterdam, S 3029-3084

Ortalo-Magné F, Prat A (2014) On the political economy of urban growth: homeownership versus affordability. Am Econ J Microecon 6:154-181

Oswald AJ (1996) A conjecture on the explanation for high unemployment in the industrialized nations: part I. University of Warwick Working Paper, Bd. 475 (December)

Oswald AJ (1997) Thoughts on NAIRU. J Econ Perspect 11:227-228

Rohe W, Stewart L (1996) Home ownership and neighborhood stability. Hous Policy Debate 7:37-81

Rosen S (1974) Hedonic prices and implicit markets: product differentiation in pure competition. J Polit Econ 82:34-55

Rossi PH, Weber E (1996) The social benefits of homeownership: empirical evidence from national surveys. Hous Policy Debate 7:1-36

Shilling JD, Sirmans CF, Dombrow JF (1991) Measuring deprecation in single-family rental and owneroccupied housing. J Hous Econ 1:368-383

Voigtländer M (2009) Why is the German homeownership rate so low? Hous Stud 24:355-372

Voigtländer M (2019) Homebuyers, liquidity constraints and private market solutions-European best practices. Intereconomics 54:53-58

Zumbro T (2014) The relationship between homeownership and life satisfaction in Germany. Hous Stud 29:319-338 